\title{
Evidence of mild founder LMOD3 mutations causing nemaline myopathy 10 in Germany and Austria
}

Ulrich A. Schatz, MD, Simone Weiss, MD, Stephan Wenninger, MD, Benedikt Schoser, MD, Wolfgang H. Muss, PhD, Reginald E. Bittner, MD, Wolfgang M. Schmidt, PhD, Anna S. Schossig, MD, Sabine Rudnik-Schöneborn, MD, and Matthias Baumann, MD

Neurology ${ }^{\circledR}$ 2018;91:e1690-e1694. doi:10.1212/WNL.0000000000006428

\section{Abstract}

\section{Objective}

To expand the clinical and genetic spectrum of nemaline myopathy 10 by a series of Austrian and German patients with a milder disease course and missense mutations in LMOD3.

\section{Methods}

We characterized the clinical features and the genetic status of 4 unrelated adolescent or adult patients with nemaline myopathy.

\section{Results}

The 4 patients showed a relatively mild disease course. They all have survived into adulthood, 3 of 4 have remained ambulatory, and all showed marked facial weakness. Muscle biopsy specimens gave evidence of nemaline bodies. All patients were unrelated but originated from Austria (Tyrol and Upper Austria) and Southern Germany (Bavaria). All patients carried the missense variant c.1648C > T, p.(Leu550Phe) in the LMOD3 gene, either on both alleles or in trans with another missense variant (c.1004A $>$ G, p.Gln335Arg). Both variants were not reported previously.

\section{Conclusions}

In 2014, a severe form of congenital nemaline myopathy caused by disrupting mutations in LMOD3 was identified and denoted as NEM10. Unlike the previously reported patients, who had a severe clinical picture with a substantial risk of early death, our patients showed a relatively mild disease course. As the missense variant c. $1648 \mathrm{C}>\mathrm{T}$ is located further downstream compared to all previously published LMOD3 mutations, it might be associated with higher protein expression compared to the reported loss-of-function mutations. The apparent clusters of 2 mild mutations in Germany and Austria in 4 unrelated families may be explained by a founder effect.

\author{
Correspondence \\ Dr. Baumann \\ matthias.baumann@ \\ tirol-kliniken.at
}

From the Department of Human Genetics (UA.S., AS.S., S.R.), Department of Pediatrics (M.B.), Medical University Innsbruck, Austria; Department of Pediatrics, Kaiser Franz Josef Hospital, Vienna, Austria (S.W.); Friedrich-Baur-Institute, Department of Neurology, Ludwig-Maximilians-University Munich, Germany (St.W., B.S.); Institute of Human Genetics, Klinikum rechts der Isar, Technische Universität Munich, Germany (UA.S., AS.S.); Institute of Pathology, SALK-LKH and PMU (Paracelsus Medical University) Salzburg, Austria (WH.M, retired); Neuromuscular Research Department, Center for Anatomy and Cell Biology, Medical University of Vienna, Austria (RE.B., WM.S.).

Go to Neurology.org/N for full disclosures. Funding information and disclosures deemed relevant by the authors, if any, are provided at the end of the article. 
Nemaline myopathy (NEM) is a genetically heterogeneous congenital myopathy with the characteristic finding of electron-dense "nemaline bodies" in myofibers. In 2014 twenty-one patients from 14 families with a severe form of congenital NEM and disrupting mutations in LMOD3 (MIM *616112) were reported. ${ }^{1}$ This form is denoted as NEM10 (MIM \#616165). Following the first patients series, 2 further families with fatal NEM10 were published. ${ }^{2,3}$

Here we describe 4 unrelated patients with clinically, ultrastructurally, and genetically confirmed NEM10 based on 2 missense mutations within LMOD3 presenting with a hitherto undescribed milder phenotype.

\section{Methods}

We reviewed the clinical features of 4 unrelated adolescent or adult patients with nemaline myopathy. The LMOD3 mutations were detected by whole exome sequencing in patients 1 and 2 , and by next generation sequencing-based panel analysis in patients 3 and 4. Histologic stains and transmission electron microscopy were performed according to standard protocols.

\section{Results}

In contrast to previous reports, our patients showed a mild disease course with pronounced facial weakness from birth but mostly stable muscle function otherwise (figure 1 ). In patient 1 reduced fetal movements were reported. Patient 2 had a history of intrauterine growth restriction and polyhydramnios resulting in Cesarean delivery. Both patients showed an impaired neonatal respiratory adaption with resuscitation needed in patient 2. Pregnancy and delivery were normal in the remaining 2 patients. Severe weakness of facial, oral, and pharyngeal muscles was present in all patients. All had swallowing difficulties after birth making transient nasogastric tube feeding necessary in 3 of them (table). All patients had dysarthric speech. When last examined, no patient had swallowing difficulties, but chewing was still not possible in patient 2 .

Despite impaired postnatal adaptation and the presence of muscular hypotonia from birth, our patients developed remarkably well and stabilized during the first year of life. Motor development was delayed in 2 patients: independent walking was achieved at age 2 years in patient 1 and at the age of nearly 6 years in patient 2. Patients 3 and 4 walked independently from the age of 16 and 14 months, respectively. Limb function was affected in all patients with lower limb weakness and a milder distally pronounced upper limb weakness. Patient 2 was nonambulatory from age 12 due to truncal weakness and progressive thoracolumbar scoliosis. Patients 1,3 , and 4 were ambulatory at ages 18,20 , and 30 years, although patient 1 was not able to run and had difficulties in climbing stairs. Mild scoliosis was present in patient 3. No patient had a clinically relevant respiratory dysfunction; only patient 2 had a reduced forced vital capacity $(45 \%)$ in lung function tests at the age of 18 years.
Figure 1 Clinical phenotype of patients with NEM10 and LMOD3 mutations
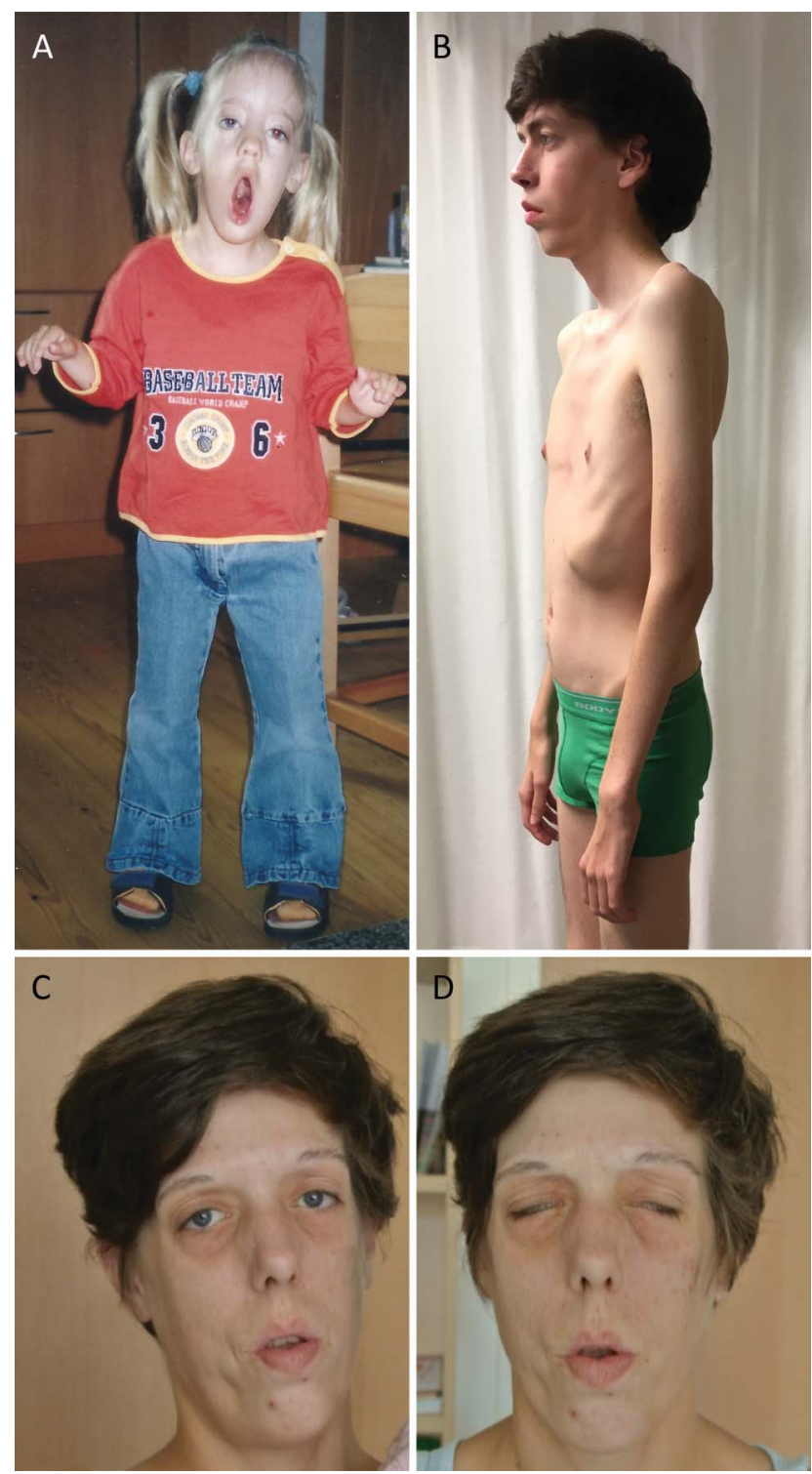

(A) Patient 2 at the age of nearly 6 years presenting with an elongated face, and severe facial and jaw weakness. (B) Patient 3 at age 20 years with a symmetrical deep funnel chest with Harrison's groove. (C and D) Patient 4 at age 30 years showing pronounced facial weakness with incomplete eye closure.

Muscle biopsy specimens showed multiple nemaline bodies in Gomori trichrome stain. Transmission electron microscopy demonstrated Z-line streaming and multiple nemaline rods (figure 2).

The patients were offspring of 4 non-consanguineous couples with Austrian (Tyrol and Upper Austria) or German (Bavaria) provenance within a maximum geographical distance of ca. $300 \mathrm{~km}$. Our patients shared 2 LMOD3 missense mutations (NM_198271.4: c.1004A $>\mathrm{G}$ and c.1648C $>\mathrm{T}$ ), either in homozygous or in compound heterozygous state (table). In all families, segregation studies were performed confirming 
Table Clinical features and genotype of the 4 patients with NEM10

\begin{tabular}{|c|c|c|c|c|}
\hline & Patient 1 & Patient 2 & Patient 3 & Patient 4 \\
\hline Age at last presentation, $y$ & 18 & 18 & 20 & 30 \\
\hline Sex & Female & Female & Male & Female \\
\hline Age at onset & Prenatal & Prenatal & Birth & Birth \\
\hline Apgar-score & $4 / 5 / 7$ & $3 / 3 / 5$ & $7 / 8 / 9$ & $8 / 9 / 9$ \\
\hline Postnatal feeding problems & + & $\begin{array}{l}+ \text { (tube feeding until age } 2 \\
\text { years) }\end{array}$ & $\begin{array}{l}+ \text { (tube feeding until age } 3 \\
\text { months) }\end{array}$ & $\begin{array}{l}+ \text { (tube feeding until age } 3 \\
\text { months) }\end{array}$ \\
\hline Age at first walking & $2 y$ & $6 y$ & $16 \mathrm{mo}$ & $14 \mathrm{mo}$ \\
\hline Ambulatory & + & - (From age $12 \mathrm{y})$ & + & + \\
\hline Limb weakness (LL > UL) & + & + & + & + \\
\hline Scoliosis & - & + (progressive) & $+($ mild $)$ & - \\
\hline $\begin{array}{l}\text { Dysarthria and pronounced facial } \\
\text { weakness }\end{array}$ & + & + & + & + \\
\hline Respiratory function & Normal (age 15 years) & $\begin{array}{l}\text { Reduced (FVC 45\%; age } \\
18 \mathrm{y})\end{array}$ & Normal (age 19 y) & Normal (age 29 y) \\
\hline LMOD3 genotype & c. $[1648 C>T] ;[1648 C>T]$ & c. $[1004 A>G] ;[1648 C>T]$ & c. $[1004 A>G] ;[1648 C>T]$ & c. $[1648 \mathrm{C}>\mathrm{T}] ;[1648 \mathrm{C}>\mathrm{T}]$ \\
\hline Provenance & Austria (Tyrol) & Austria (Upper Austria) & Germany (Bavaria) & Austria (Tyrol) \\
\hline
\end{tabular}

Abbreviations: FVC = forced vital capacity; $\mathrm{LL}=$ lower limbs; UL = upper limbs.

autosomal recessive inheritance. Both LMOD3 variants (c.1004A $>$ G, p.Gln335Arg and c.1648C > T, p.Leu550Phe) affect amino acid residues that are highly conserved up to Xenopus and zebrafish and are not listed in the in-house database (Munich Exome Server) ${ }^{4}$ nor in large reference datasets, such as the ExAC or the gnomAD browsers. In silico prediction is pathogenic according to MutationTaster, CADD, DANN, and fathmm. Both mutations are listed in ClinVar with an unknown clinical significance after detection in patient 3 .

\section{Discussion}

In contrast to the previously reported patients, who had a severe clinical picture with a substantial risk of early death, our patients showed a stable and much milder disease course. When NEM10 was genetically defined, the patients initially reported presented with intrauterine manifestations: polyhydramnios (62\%), decreased or absent fetal movements (48\%), arthrogryposis or joint contractures (48\%), or fetal edema. ${ }^{1}$ All patients had severe muscular weakness and hypotonia with a fatal outcome in most patients: 7 (33\%) died from respiratory failure in the neonatal period, 6 (29\%) patients died during the first year of life, and one pregnancy was terminated. Only 2 sisters were reported to be alive at the time of publication (age 10 and 4 years), after prenatal onset and a severe infantile disease course requiring gastrostomy and nocturnal noninvasive ventilation.

In 2017, 3 siblings with NEM10, born to consanguineous parents, due to the previously described frame-shift mutation c.138dupC, p.Ser47Glnfs ${ }^{*} 13$ were reported. ${ }^{2}$ They showed fetal hypokinesia, had congenital fractures of long bones, and died of respiratory failure during early infancy. Recently, 2 fetuses from one family have been reported with a severe prenatal disease course especially encompassing absent fetal movements and abnormal posturing. ${ }^{3}$ Genetic diagnosis of NEM10 was made after pregnancy termination with both fetuses being compound heterozygous for the previously unknown frame-shift mutation p.Glu121 Argfs ${ }^{*} 5$ and the deletion p.Leu245del in LMOD3, respectively (no transcript information has been provided for these mutations).

LMOD3 localizes mainly to the A band of the sarcomere ${ }^{5}$ and is a key regulator of thin filament length in skeletal muscle. ${ }^{1}$ It contains a tropomodulin-binding helix and 3 actin-binding domains: actin-binding helix, leucine-rich repeat domain (LRR), and Wiskott-Aldrich-syndrome protein homology 2 domain (WH2). ${ }^{1}$ Most LMOD3 mutations known so far are loss of function mutations. Protein expression analysis was performed for ten of the 15 mutations reported: protein expression was only present in 2 milder affected siblings who were compound heterozygous for 2 nonsense mutations in the $\mathrm{C}$-terminal region of the LRR domain. ${ }^{1}$ There was no protein expression for 6 other nonsense mutations; inconclusive results were reported for 2 mutations. ${ }^{1}$ Only one missense mutation (c. $976 \mathrm{G}>\mathrm{C}$, p.Gly326Arg) has been described so far. It was found in combination with 2 different nonsense mutations, causing a severe phenotype. The c.976G>C mutation lies within the LRR domain, as does the mutation c.1004A $>\mathrm{G}$ described here. 

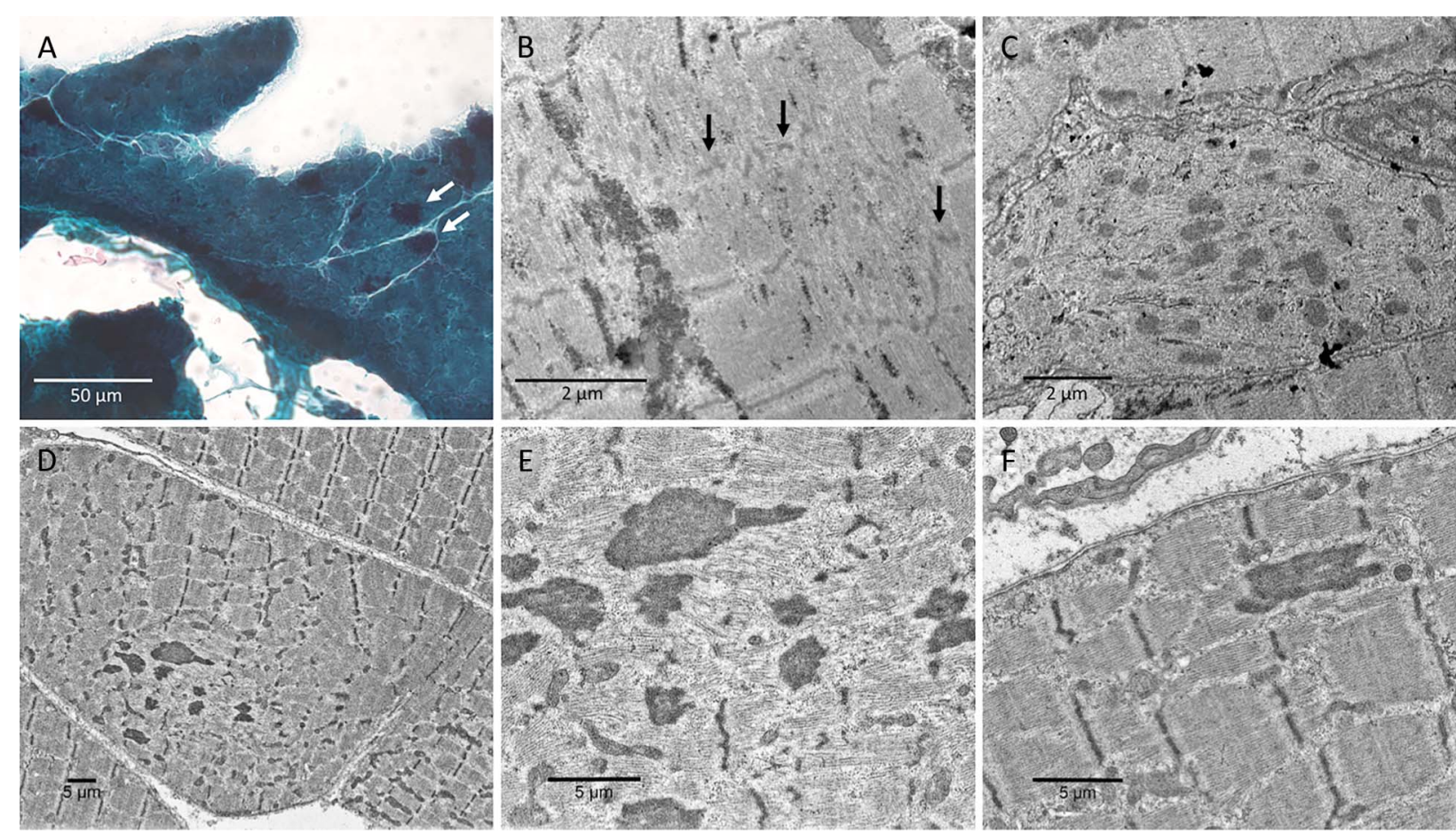

(A) Gomori trichrome stain (patient 3 at the age of 14 years) shows marked nemaline bodies (arrows) in highly atrophic myofibers. (B and C) Transmission electron microscopy images of patient 2 at the age of 3 weeks. (B) Large area of disorganized myofilaments, compatible with a core-like structure, containing dispersed Z-band material (arrows). (C) Multiple rod-bodies in a very small muscle fiber. (D-F) Transmission electron microscopy images of patient 1 at the age of 5 years. (D) Fiber displaying disturbed intrafusal filament texture and multiple electron dense nemaline rod structures. (E) Detail of rod textures out of (D). (F) Single rod-body spanning a sarcomere.

All 4 patients described in this study are homozygous or compound heterozygous for the variant $\mathrm{c} \cdot 1648 \mathrm{C}>\mathrm{T}$. This variant is located further downstream (within the $\mathrm{WH} 2$ domain) compared to all previously published LMOD3 mutations. The physicochemical difference of the affected amino acid is predicted to be small in this mutation (p.Leu550Phe) with a Grantham distance of 22, possibly leading to a more tolerable molecular change in LMOD3. The functional role for the $\mathrm{WH} 2$ domain in LMOD3 is not known to date. In LMOD1 and LMOD2 the WH2 domain acts as an actin binding domain, involved in actin nucleation, an early step in actin polymerization. ${ }^{6}$ In these 2 other LMOD proteins the equivalent to the LRR domain is the main actin binding site. The $\mathrm{WH} 2$ domain itself is responsible for a 2 to 3 -fold increase in actin nucleation activity, and mutations within the $\mathrm{WH} 2$ domain are predicted to have only a mild effect on this process. This is also supported by the observation that other domains in the C-terminal region of LMOD2 might have a stronger functional implication than the WH2 domain does. Hence, the mutation c. $1648 \mathrm{C}>\mathrm{T}$ might be responsible for the milder disease phenotype in the patients described here.

The observation of recurrent rare LMOD3 variants in patients with a clinical and morphological phenotype matching NEM supports the predictably pathogenic effect of the detected mutations. The mutational spectrum in LMOD3 remains to be further defined, but the apparent clusters of 2 mild mutations in Germany and Austria in 4 unrelated families may be explained by a founder effect.

\section{Author contributions}

U.A. Schatz: study concept and design, acquisition of data, analysis and interpretation of data, study supervision, writing and final approval of the manuscript. S. Weiss: acquisition of data, analysis and interpretation of data, critical revision of manuscript for intellectual content. S. Wenninger: acquisition of data, analysis and interpretation of data, critical revision of manuscript for intellectual content. B. Schoser: analysis and interpretation of data, critical revision of manuscript for intellectual content. W.H. Muss: acquisition of data, analysis and interpretation of data, critical revision of manuscript for intellectual content. R.E. Bittner: acquisition of data, analysis and interpretation of data, critical revision of manuscript for intellectual content. W.M. Schmidt: acquisition of data, analysis and interpretation of data, critical revision of manuscript for intellectual content. A.S. Schossig: acquisition of data, critical revision of manuscript for intellectual content. S. Rudnik-Schöneborn: acquisition of data, analysis and interpretation of data, critical revision of manuscript for intellectual content. M. Baumann: study concept and design, acquisition of data, analysis and interpretation of data, study supervision, writing and final approval of the manuscript. 


\section{Study funding}

No targeted funding reported.

\section{Disclosure}

The authors report no disclosures relevant to the manuscript. Go to Neurology.org/N for full disclosures.

\section{Publication history}

Received by Neurology March 9, 2018. Accepted in final form July 19, 2018.

\section{References}

1. Yuen M, Sandaradura SA, Dowling JJ, et al. Leiomodin-3 dysfunction results in thin filament disorganization and nemaline myopathy. J Clin Invest 2014;124:4693-4708.

2. Abbott M, Jain M, Pferdehirt R, et al. Neonatal fractures as a presenting feature of LMOD3-associated congenital myopathy. Am J Med Genet A 2017;173:2789-2794.

3. Berkenstadt M, Pode-Shakked B, Barel O, et al. LMOD3-associated nemaline myopathy: prenatal ultrasonographic, pathologic, and molecular findings. J Ultrasound Med 2018;37:1827-1833.

4. Kremer LS, Bader DM, Mertes C, et al. Genetic diagnosis of Mendelian disorders via RNA sequencing. Nat Commun 2017;8:15824.

5. Garg A, O’Rourke J, Long C, et al. KLHL40 deficiency destabilizes thin filament proteins and promotes nemaline myopathy. J Clin Invest 2014;124:3529-3539.

6. Boczkowska M, Yurtsever Z, Rebowski G, Eck MJ, Dominguez R. Crystal structure of leiomodin 2 in complex with actin: a structural and functional reexamination. Biophys J 2017;113:889-899. 